\title{
Theory Opening Talk
}

\section{Benjamín Grinstein ${ }^{* \dagger}$}

University of California, San Diego

E-mail: bgrinstein@ucsd.edu

\begin{abstract}
As a preview to the conference I first make a brief account of the methods that are used by theorists in the context of Kaon physics. I then make two modest observations. First, that looking at nonKaon physics may shed light on Kaon physics. As a case in point I argue that one can empirically find analogues of the $\Delta I=1 / 2$ rule in both $D$ and $B$ decays to two pseudoscalars. And second, that while in tests of fundamental principles the scale of putative new physics is often unknown a priori, there are some cases where it is known. As an example I discuss tests of Lorentz invariance and argue that, in the context of Doubly Special Relativity, the $K^{0}-\bar{K}^{0}$ mass difference is already sensitive to Planck scale effects.
\end{abstract}

2013 Kaon Physics International Conference,

29 April-1 May 2013

University of Michigan, Ann Arbor, Michigan - USA

\footnotetext{
* Speaker.

$\dagger$ The author wishes to thank the organizers of KAON2013 for partial support to attend the conference. Work supported in part by the US DOE under contract DE-SC0009919.
} 


\section{Introduction}

After more than half a century since the discovery of the Kaon there remain many interesting and difficult questions facing theorists. Why is this so? Why is Kaon theory hard? There is little question that QCD is responsible for the underlying dynamics. So it may be instructive to review how progress has been made in solving QCD dynamics:

1. At very short distance or time scales the interaction is very weak. This is a manifestation of asymptotic freedom. For processes that involve very short time scales compared to the typical hadronization time, $\sim 10^{-25} \mathrm{~s}$, theorists may reliably use perturbation theory. For example, since the lifetime of the top quark is much shorter than the confinement scale we use perturbation theory to reliably compute its production rate, its width and inclusive decay branching fractions.

2. When several time scales are involved in a process one can frequently factorize an amplitude into (a convolution of) short and long distance/time scale terms. The short distance "hard" terms are computable perturbatively and are process dependent. The long distance terms are not amenable to treatment via perturbation theory, but are universal in the sense that they are common to many different processes. This universality allows theorists to relate processes even without being able to compute the long distance contributions to the amplitudes. Examples abound. In deep inelastic scattering one factorizes production rates into long distance parton distribution functions and hard scattering partonic cross sections. SCET and HQET are effective field theories based on the factorization of heavy hadron amplitudes into a long distance jet and soft functions (SCET) or Isgur-Wise functions (HQET) and hard kernels. Even in weak interactions the effective Fermi-like theory is a factorization into short distance Wilson coefficients and long distance matrix elements of operators.

The reason $K$-physics is hard is that all energy scales are soft (there are no short time scales). Neither perturbation theory nor factorization are useful. The latter has a double problem: not only is there no hard amplitude but the variety of processes is small so the universality of would-be long distance factorized terms would be of little use.

It is no surprise that the tools we use for $K$-theory are different. It is through a combination of symmetry arguments, lattice simulations of QCD and chiral Lagrangians that we have some handle on this problem. These are tightly inter-related. Chiral Lagrangians are, of course, a practical implementation of soft pion theorems that are based on symmetry. And lattice calculations profit from the formulation in terms of chiral Lagrangians, just as the results of lattice calculations inform the chiral Lagrangian formulation.

Let's look at a couple of examples of this symbiotic relation, first of symmetry and lattice. Description of $K_{\ell 3}$ decays require knowledge of form factors $f_{+, 0}$ defined by

$$
\left\langle\pi\left(p^{\prime}\right)\left|V^{\mu}\right| K(p)\right\rangle=f_{+}\left(q^{2}\right)\left(p+p^{\prime}-\frac{m_{K}^{2}-m_{\pi}^{2}}{q^{2}} q\right)^{\mu}+f_{0}\left(q^{2}\right) \frac{m_{K}^{2}-m_{\pi}^{2}}{q^{2}} q^{\mu},
$$

where $V^{\mu}$ is a vector current, $q=p-p^{\prime}$ and from kinematics $f_{+}(0)=f_{0}(0)$. Now, $S U(3)$ symmetry gives $f_{+}\left(q^{2}\right)=f_{0}\left(q^{2}\right)$ and $f_{+}(0)=1$. If nature is close to the symmetry limit, measurement of the spectrum of $K_{\ell 3}$ decay leads then to a precise determination of the Cabibbo angle. It is therefore important to determine deviations from the symmetry limit prediction $f_{+}(0)=1$. The AdemolloGatto theorem shows that if $\delta$ is the small parameter that characterizes the departures from the 
symmetry limit, say $\delta \sim 20 \%$ for $S U(3)$, then deviations linear in $\delta$ from this limit for $f_{+}(0)$ are of order $\delta^{2}: f_{+}(0)=1+0 \cdot \delta+\mathscr{O}\left(\delta^{2}\right)[1]$. While $\left|f_{+}(0)-1\right| \lesssim \delta^{2} \sim 4 \%$ is very good, particularly given the small effort required, it is today not good enough, given the experimental precision with which these decays are measured. For further progress we turn to lattice computations. This give a remarkably precise determination, $f_{+}(0)=0.9667 \pm 0.0023 \pm 0.0033$ [2]. Note not only that this is consistent with expectations from the Ademollo-Gatto theorem but also that the computation benefits from it: it is a determination of $4 \%$ corrections with $10 \%$ accuracy!

Now for an example involving chiral Lagrangians. $\varepsilon^{\prime} / \varepsilon$, that characterizes CP-violation in direct $K \rightarrow \pi \pi$ decays, is smaller than naively expected because a cancellation of competing QCD and electroweak penguin contributions [3]. We pointed out a long time ago that it is possible that this accidental cancellation is absent in direct CP-violation in $K \rightarrow \pi \pi \pi$ decays [4]. A recent re-analysis gives[5]

$$
\begin{aligned}
\operatorname{Re}\left(\varepsilon^{\prime} / \varepsilon\right) & =-(1.88 \pm 1.0) \operatorname{Im} G_{8}-(0.38 \pm 0.13) \operatorname{Im}\left(e^{2} G_{E}\right) \\
10^{2} \Delta g & =(0.7 \pm 0.1) \operatorname{Im} G_{8}-(0.07 \pm 0.02) \operatorname{Im}\left(e^{2} G_{E}\right)
\end{aligned}
$$

where $\Delta g$ is the slope asymmetry, with the slope defined as in

$$
\frac{\left|A_{K^{+} \rightarrow 3 \pi}\left(s_{1}, s_{2}, s_{3}\right)\right|^{2}}{\left|A_{K^{+} \rightarrow 3 \pi}\left(s_{0}, s_{0}, s_{0}\right)\right|^{2}}=1+g y+\mathscr{O}\left(y^{2}, x^{2}\right), \quad x \equiv \frac{s_{1}-s_{2}}{m_{\pi}^{2}}, y \equiv \frac{s_{3}-s_{0}}{m_{\pi}^{2}} .
$$

Here $G_{8}$ and $G_{E}$ characterize the QCD and electroweak penguin contributions in the chiral Lagrangian. I have omitted terms in $\Delta g$ from higher derivative counterterms to the chiral Lagrangian that do not contribute to $\varepsilon^{\prime} / \varepsilon$. In principle a precise measurement of the 3-body decay spectrum, or simulations of QCD on the lattice, can determine several independent combinations of coefficients in the chiral Lagrangian and hence predict $\varepsilon^{\prime} / \varepsilon$ and $\Delta g$.

So while the popular perturbative or factorization methods of QCD are not directly applicable to Kaon physics, we do have powerful tools at our disposal. And that's good news since $K$ physics offers unique opportunities: (i) it is clean, e.g., $K \rightarrow \pi \pi$ vs $K \rightarrow \pi \pi \pi$ (ii) high statistics, e.g., $4 \times 10^{9} K^{+} \rightarrow \pi^{+} \pi^{+} \pi^{-}$at NA48/2, (iii) it offers fundamental tests, e.g., of $T$-violation, $C P T$ violation, Quantum Mechanics (coherence), (iv) strongly constraints the parameters of the SM and tests the consistency of the CKM model, its accounting of CP-violation and universality, and (v) it provides exquisite indirect tests of New Physics, e.g., in $K \rightarrow \pi v v$.

In the rest of this conference (or now, proceedings volume) I look forward to learn much more about the state of the art of all this. But in the rest of this talk I advance a couple of original thoughts.

\section{2. $\Delta I=1 / 2$ rule revisited}

The first modest proposal is this: perhaps we can learn about dynamics of kaons by looking outside the realm of $K$-physics, like looking under the lamppost. In particular, we may either learn from, or challenge what we think of as good understanding, by thinking about $D$ or $B$ mesons.

Case in point: the $\Delta I=1 / 2$ rule, that states that empirically the amplitude for $K \rightarrow \pi \pi$ with pions in an $I=0$ state is enhanced relative the $I=2$ state:

$$
\frac{\operatorname{Re} A_{0}}{\operatorname{Re} A_{2}}=22.5 \text {. }
$$


We have no good theoretical explanation for this. The lattice is making great strides towards reproducing this number; see, e.g., Ref. [6]. But is this producing understanding of the mechanism that produces the large number? Lattice theorists believe it does [7], and I am not about to criticize those arguments. Instead I will challenge them. The basis for this is that the latticist's explanation is, by her own account, very specific to Kaon decay, and she will therefore not expect to see such an enhancement in the decay of other mesons, like the $D$ or $B$-mesons.

Really? Let's put that to the test. ${ }^{1}$ Consider first $D^{0} \rightarrow K^{+} K^{-} / \pi^{+} \pi^{-}$. Assuming $S U(3)-$ symmetry one finds for the amplitudes[8]

$$
\begin{aligned}
& \mathscr{A}\left(D^{0} \rightarrow K^{+} K^{-}\right)=(2 T+E-S) \Sigma+\frac{1}{2}(3 T+2 G+F-E) \Delta \\
& \mathscr{A}\left(D^{0} \rightarrow \pi^{+} \pi^{-}\right)=-(2 T+E-S) \Sigma+\frac{1}{2}(3 T+2 G+F-E) \Delta
\end{aligned}
$$

where $\Sigma \equiv \frac{1}{2}\left(V_{c s}^{*} V_{u s}-V_{c d}^{*} V_{u d}\right)$ and $\Delta \equiv \frac{1}{2}\left(V_{c s}^{*} V_{u s}+V_{c d}^{*} V_{u d}\right) . S, E$ and $F$ are the invariant matrix elements between a $D$ mesons and a meson pair in an octet of the $\overline{\mathbf{6}}, \mathbf{1 5}$ and $\mathbf{3}$ components of the weak Hamiltonian, respectively, $G$ of the $\mathbf{3}$ to a singlet pair and $T$ of the $\mathbf{1 5}$ to a meson pair in the 27. Note that $\Sigma \approx \lambda=\sin \theta_{C}$, while $|\Delta| \sim \lambda^{5}$, so that $|\Delta| / \Sigma \sim 10^{-3}$. Neglecting $\Delta$ one would have $\Gamma\left(D^{0} \rightarrow K^{+} K^{-}\right)=\Gamma\left(D^{0} \rightarrow \pi^{+} \pi^{-}\right)$in the $S U(3)$ limit. Experimentally $\Gamma\left(D^{0} \rightarrow K^{+} K^{-}\right) / \Gamma\left(D^{0} \rightarrow\right.$ $\left.\pi^{+} \pi^{-}\right) \approx 3$ requires both the $\Sigma$ and $\Delta$ terms in the amplitude to contribute with similar strengths. Barring accidental cancellations this means that the matrix elements $G$ and $F$ are significantly enhanced. Since $\Delta$ has a large phase, we predicted significant CP-violation in these decays [9].

Of course, $S U(3)$-breaking can play a significant role. Additional contributions to the amplitudes, suppressed by $\delta(S U(3)$-breaking) but not by $\Delta$ can enter in distinct combinations that may help explain $\Gamma\left(D^{0} \rightarrow K^{+} K^{-}\right) / \Gamma\left(D^{0} \rightarrow \pi^{+} \pi^{-}\right) \approx 3$ without such a large enhancement of $G$ and $F$. Fitting to the now observed, surprisingly large, $\mathrm{CP}$-asymmetries in these decays, in addition to the decay rates, including effects linear in $\delta$, it is found that $F$ and $G$ still are enhanced, but "only" by a factor of between 10 and 50 [10]. The enhancement in $F$ and $G$ is similar to that of the $\Delta I=1 / 2$ rule in that it appears in matrix elements of the smallest $S U$ (3)-representation of the Hamiltonian. In this case it is $\mathbf{3}$ (as opposed to the $\overline{\mathbf{6}}$ and $\mathbf{1 5}$ ), while for the $\Delta I=1 / 2$ rule the dominant piece is from the $I=1 / 2$ Hamiltonian (as opposed to the $I=3 / 2$ one).

We are conducting an investigation of similar effects in $B$ decays, in particular $B \rightarrow K \pi$ [11]. Assuming isospin, the Hamiltonian is either $I=0$ or $I=1$ and the final $K \pi$ is either an $I=\frac{1}{2}$ or $\frac{3}{2}$ state. Denote invariant matrix elements by

$$
\langle\mathbf{2}|\mathbf{1}| B\rangle=P_{b},\left\langle\mathbf{2}\left|\mathbf{1}^{\prime}\right| B\right\rangle=P_{a},\langle\mathbf{2}|\mathbf{3}| B\rangle=T,\langle\mathbf{4}|\mathbf{3}| B\rangle=S .
$$

We have split the singlet contribution into $P_{b}$ which appears from the tree level Hamiltonian and $P_{a}$ which arises from a one loop penguin. This is so that we may keep track of the different combination of CKM matrix elements that arises at one loop. A fit to both CP-conserving and CP-violating observables gives a marked enhancement of the singlet contributions. The (preliminary!) best fit gives $P_{a} / P_{b} \simeq 12, P_{a} / S \simeq 17, P_{a} / T \simeq 48$. The fit includes a parameter $x$ that measures the weight of the annihilation contribution relative to all others; the expectation is that $x \sim \frac{\alpha_{s}}{8 \pi}\left|\frac{V_{t b}^{*} V_{t s}}{V_{u b}^{*} V_{u s}}\right| \approx 0.25$ and the fit value is very close to this. It is apparent that also in $B$ decays there is an enhancement of the low isospin contribution relative the higher isospin ones!

\footnotetext{
${ }^{1}$ To be sure, my work on amplitude enhancement in $B$ decays was not prompted by these new lattice results on the $\Delta I=1 / 2$ rule. In fact, much of it preceded it.
} 


\section{CPT/QM-like bounds}

Kaon physics is a good place to test the validity of some of our most cherished principles. One may check, for example, whether CPT is a good symmetry [12]. We believe it is because of the CPT theorem that states that any local, unitary QFT is invariant under CPT. The assumptions of the CPT theorem are not satisfied by theories of Quantum Gravity, like string theory or loop-QG, that are non-local. Alternatively, black holes do not carry discrete charge, erasing the CPT quantum number of stuff that falls into them. However, it is known that CPT is conserved in these. So while testing for CPT is interesting, we do not know a priori what is the scale of new physics associated with the putative violation of CPT.

A similar argument can be made about possible deviations from the axioms of Quantum Mechanics (QM) [13]. In QM pure states do not evolve into mixed states. Because of black holes information loss Hawking proposed a generalization of QM which allows evolution of pure states into to mixed ones [14]. Page showed that this leads to CPT violation [15]. The consensus now seems to be, however, that pure states do not evolve into mixed ones in a quantum theory of gravity. So again, testing the validity of $\mathrm{QM}$ does not give us a priori a scale associated with the new physics. This is true also in some more ad hoc formulations of beyond-QM, like Weinberg's non-associative matrix QM [16] or Eberhard's test of the existence of a unitary $S$-matrix [17].

My second modest proposal is that tests of the validity of Lorentz invariance can be associated with a definite scale of new physics, namely, the Planck scale. And, moreover, that in some cases tests from Kaon physics are as sharp as the more conventional ones form interferometry. The latter are based on the extension of the QED part of the SM by a Lorentz violating term, as follows [18]:

$$
\mathscr{L}=-\frac{1}{4} F^{\mu v} F_{\mu v}-\frac{1}{4} k_{\mu v \lambda \sigma} F^{\mu v} F^{\lambda \sigma} .
$$

Here $k$ is a set of non-dimensional constants, organized as a tensor but inert under Lorentz transformations, that characterize the violations of Lorentz symmetry. These describe propagation of EM waves in an anisotropic medium:

$$
\left(\begin{array}{l}
\vec{D} \\
\vec{H}
\end{array}\right)=\left(\begin{array}{cc}
1+\kappa_{D E} & \kappa_{D B} \\
\kappa_{H E} & 1+\kappa_{H B}
\end{array}\right)\left(\begin{array}{l}
\vec{E} \\
\vec{B}
\end{array}\right)
$$

with $\left(\kappa_{D E}\right)^{j k}=-2(k)^{0 j 0 k},\left(\kappa_{H B}\right)^{j k}=\frac{1}{2} \varepsilon^{j p q} \mathcal{E}^{k r s}(k)^{p q r s}$ and $\left(\kappa_{D B}\right)^{j k}=-\left(\kappa_{H E}\right)^{k j}=-2(k)^{0 j p q} \mathcal{E}^{j p q}$. Some linear combinations of these, that produce bi-refringence, are bound at some ridiculously low level, $10^{-32}$ or so. Beautiful experiments using a modern version of the Michelson-Morley interferometer place bounds on some combination of the anisotropic medium parameters that do not produce bi-refringence of order of a few parts in $10^{-18}[19,20]$.

What may be the origin of Lorentz violation, and what is the scale associated with it? There are many proposals. Doubly Special Relativity (DSR) poses that in addition to the speed of light being boost invariant there is an invariant length scale, the Planck Length, or equivalently an invariant energy, the Planck mass. In non-commutative spacetime theory the position operator is taken to satisfy $\left[x^{\mu}, x^{v}\right]=\theta^{\mu v}$. The dimensional parameter $\theta^{\mu v}$ sets the scale of Lorentz symmetry violation and is taken to be the appropriate power of the Planck Length. And so on with many others, like rainbow metric, $\kappa$-Minkowski space-time, Hopf-algebras, space-time foam, etc. 
I will concentrate on one example, namely, DSR. For a (non-unique) implementation of DSR you construct a non-linear realization of the Lorentz group. That is, consider a map $F: P \rightarrow \mathscr{P}$ from the space of physical four-momentum $P=\left\{\left(p^{0}, \vec{p}\right)\right\}$ to a fictitious, but linear space $\mathscr{P}=$ $\left\{\left(\pi^{0}, \vec{\pi}\right)\right\}$. Then define the Lorentz transformation on physical states, in terms of the familiar linear Lorentz transformation $\Lambda$ on the fictitious states, by $p^{\prime}=F^{-1}(\Lambda F(p))$. Taking now a map such that $F\left(p_{P}\right)=0$ (or infinity), where $p_{P}$ is a special momentum such as $p^{0}=\kappa$, then this special momentum will remain boost invariant. For example one may have

$$
E^{\prime}=a\left(c E+s p_{1}\right), p_{1}^{\prime}=a\left(s E+c p_{1}\right), p_{2}^{\prime}=a p_{2}, p_{3}^{\prime}=a p_{3}
$$

where $a^{-1}=1+\kappa^{-1}\left[(c-1) E+s p_{1}\right], c=\cosh \xi$ and $s=\sinh \xi$. This gives the dispersion relation

$$
E^{2}-\vec{p}^{2}=m^{2}(1-E / \kappa)^{2} .
$$

At high energy this gives

$$
E \approx p+\frac{m^{2}}{p}-\frac{1}{2} \frac{E^{2}}{\kappa}
$$

which can also be taken as a parametrization of a wide class of implementations of DSR. You cannot fail to notice that this gives an energy dependent speed of light! This leads, for example, to energy dependent time delays in arrival of signals from Gamma-Ray-Bursts (GRB), $\Delta t \approx(\Delta E / \kappa) L$. Analysis of GRB data puts a bound $\kappa>1.3 \times 10^{18} \mathrm{GeV} \approx 0.1 M_{\text {Planck }}$ [21].

What is remarkable is that Kaon physics places a similar bound. Kaon physics is sensitive to the Planck scale! To see this we really need a QFT of DSR. We don't have this. Instead we consider the generalized Klein-Gordon equation based on the dispersion relation (3.4) [22]. The energies are given by

$$
E=\frac{-m^{2} / \kappa \pm \sqrt{\left(1-m^{2} / \kappa^{2}\right) \vec{p}^{2}+m^{2}}}{1-m^{2} / \kappa^{2}}
$$

A non-relativistic expansion allows us to find the mass of the particle and hole, interpreted as the anti-particle (the two signs in (3.6)):

$$
m^{ \pm}=\frac{m}{1 \pm m / \kappa}
$$

The best bound on particle-antiparticle mass difference $\Delta m$ is from Kaon physics. It gives

$$
\kappa>\frac{2 m^{2}}{(\Delta m / m)} \approx 1.1 \times 10^{18} \mathrm{GeV} .
$$

Coincidentally this is the same bound as that from GRB described above.

\section{Conclusions}

You may find this talk far from what you expected for an opening talk for this conference. You may have expected perspective, the field in re(pre)view. But this is a special year. The higgs was discovered, but nothing else, and except for few pesky but inconclusive deviations - like the Tevatron's $t \bar{t}$-FB asymmetry and $g-2$ of the muon - it looks like the SM is in good shape. 
But Kaon physicists knew this all along. More generally, flavor physicists knew this all along. Generic bounds on new physics (NP) place the presumed scale of NP well outside the reach of the LHC. In particular CP-violation in kaon mixing, parametrized by a local four-fermion interaction characterized by a NP scale $\Lambda$ gives a generic bound $\Lambda>10^{4} \mathrm{TeV}$. Even with Minimal Flavor Violation the bound is a hefty $\Lambda>10 \mathrm{TeV}$. The importance of new $K$-tests of the standard paradigm cannot be overstated nor is the importance of improved precision in classical tests/measurements on Kaon physics. Looking forward to a fruitful and exciting conference!

\section{References}

[1] M. Ademollo and R. Gatto, Phys. Rev. Lett. 13, 264 (1964).

[2] A. Bazavov et al., arXiv:1212.4993 [hep-lat].

[3] J. Bijnens and M. B. Wise, Phys. Lett. B 137, 245 (1984).

[4] B. Grinstein, S. -J. Rey and M. B. Wise, Phys. Rev. D 33, 1495 (1986).

[5] J. Prades, E. Gamiz and I. Scimemi, Nucl. Phys. Proc. Suppl. 164, 79 (2007) [hep-ph/0509346].

[6] T. Blum, P. A. Boyle, N. H. Christ, N. Garron, E. Goode, T. Izubuchi, C. Jung and C. Kelly et al., Phys. Rev. Lett. 108, 141601 (2012) [arXiv:1111.1699 [hep-lat]].

[7] P. A. Boyle et al. [RBC and UKQCD Collaborations], arXiv:1212.1474 [hep-lat].

[8] C. Quigg, Z. Phys. C 4, 55 (1980).

[9] M. Golden and B. Grinstein, Phys. Lett. B 222, 501 (1989).

[10] D. Pirtskhalava and P. Uttayarat, Phys. Lett. B 712, 81 (2012); B. Bhattacharya, M. Gronau and J. L. Rosner, Phys. Rev. D 85, 054014 (2012); H. -Y. Cheng and C. -W. Chiang, Phys. Rev. D 86, 014014 (2012); J. Brod, Y. Grossman, A. L. Kagan and J. Zupan, JHEP 1210, 161 (2012); T. Feldmann, S. Nandi and A. Soni, JHEP 1206, 007 (2012)

[11] B. Grinstein, D. Pirtskhalava, D. Stone and P. Uttayarat, in preparation

[12] P. Huet and M. E. Peskin, Nucl. Phys. B 434, 3 (1995) [hep-ph/9403257].

[13] J. R. Ellis, N. E. Mavromatos and D. V. Nanopoulos, Phys. Lett. B 293, 142 (1992) [hep-ph/9207268].

[14] S. W. Hawking, Phys. Rev. D 14, 2460 (1976).

[15] D. N. Page, Gen. Rel. Grav. 14, 299 (1982).

[16] S. Weinberg, Phys. Rev. Lett. 62, 485 (1989).

[17] P. Eberhard, "Should unitarity be tested experimentally?," CERN-72-01; W. C. Carithers el al, Phys. Rev. D 14, 290 (1976).

[18] D. Mattingly, Living Rev. Rel. 8, 5 (2005) [gr-qc/0502097].

[19] S. Herrmann et al, Phys. Rev. D 80, 105011 (2009) [arXiv:1002.1284 [physics.class-ph]].

[20] C. .Eisele, A. Y. .Nevsky and S. Schiller, Phys. Rev. Lett. 103, 090401 (2009).

[21] G. Amelino-Camelia and L. Smolin, Phys. Rev. D 80, 084017 (2009) [arXiv:0906.3731 [astro-ph.HE]].

[22] M. Coraddu and S. Mignemi, Europhys. Lett. 91 (2010) 51002 [arXiv:0911.4241 [hep-th]]. 